\title{
Una indagación narrativa en los procesos de construcción curricular a partir de relatos de experiencias de docentes en ciernes durante la formación del profesorado
}

\author{
Luis Gabriel Porta \\ Universidad Nacional de Mar del Plata, CONICET \\ mail: luisporta510Agmail.com \\ ORCID: https://orcid.org/0000-0002-5828-8743
}

\author{
María Cristina Sarasa \\ Universidad Nacional de Mar del Plata \\ mail: m.cristina.sarasaQdgmail.com \\ ORCID: https://orcid.org/0000-0003-0849-2911
}

\section{RESUMEN}

Este trabajo constituye una indagación narrativa como pedagogía narrativa sobre experiencias formativas en el currículum del profesorado de inglés. Allí transcurren las vidas estudiantiles, cuyos relatos co-compuestos negocian significados vivenciales. Nos inscribimos en una ontología curricular del devenir y de la experiencia en el contexto de la formación inicial del profesorado de inglés en una universidad nacional argentina, con autores-investigadores participantes y 24 estudiantes. La pregunta central gira en torno a cómo se entraman las narrativas estudiantiles sobre la buena enseñanza durante su formación. Definimos al currículum como una co-construcción donde sus protagonistas crean conocimientos a partir de sus vidas. Prestarles cuidado ontológico, otorgarles respeto epistemológico y brindarles atención ética a esas existencias requieren curricula sensibles a las vidas y las experiencias relatadas. Metodológicamente, la indagación narrativa como pedagogía narrativa nos permitió urdir hebras textuales sobre la buena docencia expresadas en relatos co-narrados con los estudiantes. De nuestros entramados narrativos surgen experiencias vividas sobre el s(ab) er de la buena docencia. Concluimos elucidando las implicancias de nuestros relatos, mientras sostenemos la recuperación pública de experiencias íntimas sobre vivencias silenciadas acerca de qué es ser buen docente en los curricula de su formación.

Palabras Clave: Formación inicial del profesorado; currículum; indagación narrativa; pedagogía narrativa; buena docencia.

\section{A narrative inquiry into curriculum-construction processes through future teachers' experiential stories during their initial education}

\section{ABSTRACT}

This paper engages in a narrative inquiry as narrative pedagogy into formative experiences in English teacher education curricula. These constitute sites where students' lives unfold and are expressed through co-composed texts negotiating meanings of their storied experiences. As authors and participant researchers, we embrace a curricular ontology of becoming and feeling with 24 undergraduates in the context of their initial English-language teacher education in an Argentinean state university. Our research question concerns the ways in which our students' narratives on good teaching become intertwined and emplotted during their education. We define curriculum as a co-construction whose protagonists generate personal practical knowledge. It deserves ontological care, epistemological respect, and engagement in relational ethics manifested in curricular sensitivity towards storied lives and experiences. Narrative inquiry as narrative pedagogy allowed us to interweave narrative strands about good teaching expressed in the 24 students' co-narrated stories. Embodied understandings about living - as well as knowing about-good teaching emerge from these storied threads. We conclude by discussing the implications of our narratives as they publicly retrieve intimate, often silenced, experiences about the meanings of good teaching in initial formation curricula.

Keywords: Initial teacher education; curriculum; narrative inquiry; narrative pedagogy; good teaching.

ISSN: 0210-2773

DOI: https://doi.org/10.17811/rifie.50.3.2021.721-728 


\section{Introducción}

La otrora racionalidad técnica en los curricula para la formación del profesorado ha cedido el paso a espacios que ponen en juego la continuidad temporal de las experiencias de aprendizaje de futuros docentes. En este contexto, las vivencias que cada docente en ciernes ha sostenido sobre la buena enseñanza y sus buenos profesores le atañe personal y profesionalmente (Stenberg, Karlsson, Pitkaniemi, \& Maaranen, 2014). La narrativa permite entrelazar los vínculos entre la buena enseñanza, los conocimientos docentes y la formación del profesorado.

Para abordar la expresión de las vivencias relatadas, la indagación narrativa se concentra en interpretar a la experiencia como fuente de conocimiento (Clandinin, 2016). Mantiene un compromiso con el curriculum vivido de la formación docente en sus aulas, donde esta experiencia se co-construye mediante la pedagogía narrativa. Ésta implica una práctica narrativa durante la cual formadores de docentes y futuros docentes viven, cuentan, vuelven a vivir y vuelven a contar, en este caso, sus relatos de buena enseñanza y buenos docentes (Huber, Caine, Huber, \& Steeves, 2014; Seiki, Caine, \& Huber, 2018). El propósito central de la indagación narrativa constituye, entonces, un enfoque pedagógicamente orientado a la práctica narrativa reflexiva. De esta manera, la motivación que subyace a nuestra exploración como investigadores-profesores implicados en nuestro contexto universitario es la idea de compartir sitios donde docentes en formación puedan aprender con sus relatos de buena enseñanza y buenos profesores en lugar de hacerlo rutinariamente acerca de teorías, experticias ajenas, manuales, textos, o relatos ajenos. Nuestro interrogante principal aborda, entonces, las formas en que se entraman experiencias vividas en, y con, la buena docencia mediante relatos compartidos durante la formación inicial de 24 participantes-estudiantes que transitan el profesorado de inglés en una universidad nacional argentina.

El trabajo resume tradiciones críticas en el campo del currículum (ej. Apple, 1986; Liston \& Zeichner, 1997; Popkewitz, 2010) para luego efectuar un giro ontológico en el currículum de la formación del profesorado, relacionándolo con la experiencia vivida (Dewey, 1998; Magrini, 2017). Esta orientación nos ubica en el currículum de vidas (Rosiek \& Clandinin, 2016) donde, en nuestro caso, recuperamos al conocimiento práctico personal que los estudiantes del profesorado relatan acerca de la buena docencia y los buenos docentes (Clandinin, 2019). Metodológicamente, desde estas inscripciones conceptuales, la indagación narrativa como pedagogía narrativa (Huber et al., 2014) nos permitió recoger y urdir hebras textuales expresadas en relatos sobre buena enseñanza co-narrados en el aula con nuestros 24 estudiantes. De estos entramados narrativos 'densos', que nuestro trabajo entreteje con las locuciones de las voces estudiantiles (Caine \& Steeves, 2009), brotan experiencias vividas sobre el s(ab)er de la buena docencia y los buenos docentes. Finalizamos dilucidando las implicancias emergentes de nuestras tramas relatadas para el currículum de la formación inicial del profesorado, mientras sostenemos nuestra recuperación pública de conocimientos y devenires íntimos en torno a vivencias a menudo silenciadas acerca de qué es ser buen docente en los currícula de su educación.

\section{Múltiples sentidos del campo del currículum}

Hace más de medio siglo que el campo del currículum experimentó una vasta resignificación con la publicación de La vida en las aulas (Jackson, 1968). La noción del currículum oculto develó sus profundidades subyacentes, en tanto las escuelas hacen algo más que transmitir conocimiento neutro al definirlo y certificar- lo. Mientras éstas fundan socialmente sus procesos de enseñan$\mathrm{za}$, los estudiantes aprenden 'cosas' que no figuran explícitamente en el currículum formal. La obra habilitó una tradición crítica en los estudios sobre el currículum, ya que éste plasma ideas normativas de legitimación de valores y culturas. El conocimiento escolar formal y el currículum oculto recrean la hegemonía, modelos fundamentales de la sociedad mantenidos por suposiciones ideológicas tácitas o normas inconscientes, así como por el ejercicio del poder y del control económico (ej. Apple, 1986).

Esta vía crítica fue revelando otros currícula. El currículum nulo indica procesos de exclusión de conocimientos y prácticas sociales que han perdido su lucha por conseguir legitimidad (Eisner, 1994). Éste se puebla de violencia simbólica, expresada en el monopolio del poder de asignar instrumentos de conocimiento y de expresión (Bourdieu, 2006). En las batallas por la apropiación de significados curriculares y la producción de sentidos, ocurren traducciones pedagógicas que operan alquimias en los traspasos del conocimiento disciplinar al conocimiento curricular (Popkewitz, 2010). Ya sea más estructural (De Alba, 1998) o más personal, 'de' docentes y 'de lo que' estudiantes hacen con lo que reciben del currículum 'de' docentes (Gimeno Sacristán, 1998), el currículum no deja de ser bancario, en cuanto suele efectuar una donación de conocimientos hacia estudiantes que se presume ignorantes de los contenidos a impartir (Freire, 1970).

La formación docente ha abrevado en estos currícula, recurriendo a la metáfora deportiva del nadador abrumado por teorías aprendidas fuera del agua, incapaz de desplazarse en el mar embravecido de la práctica (Santos Guerra, 2008). Paralelamente, la corriente crítica había señalado a la reproducción, la a-criticidad, la práctica irreflexiva y las teorías vacías en la formación (Liston \& Zeichner, 1997). No obstante, persisten las trazas de la bancarización, la donación y la recepción, las tradiciones, las alquimias y la violencia simbólica. Respecto del conocimiento docente para enseñar currícula normados, aún reina en muchos sitios de formación docente el paradigma del conocimiento pedagógico del contenido (Shulman, 1986).

Sin embargo, es posible indagar sobre el ser en la educación, otorgarle un giro ontológico al currículum cuando visualizamos a la clase de formación del profesorado como un lugar para habitar y ser-junto-con los demás (Magrini, 2017). Así, podemos enriquecer la noción del currículum como "lugar, espacio, territorio... trayectoria, viaje, recorrido... autobiografía, nuestra vida, currículum vitae. En el currículum se forja nuestra identidad. El currículum es texto, discurso, documento... es documento de identidad" (Da Silva, 2001, p. 187).

Mediante esta reconceptualización, que nos acerca a un plano ontológico relacionado con la experiencia vivida (Dewey, 1998), y a uno epistemológico relacionado con el conocimiento narrativo de esa experiencia (Clandinin, 2016), nos aproximamos al currículum de vidas. Éste resulta un viaje o transcurso vital experiencial de seres que, en sus rutas de aprendizaje, co-construyen conocimiento a partir de sus existencias. Este currículum no puede ser generalizador, ni generalizable, porque cada protagonista-estudiante y docente-vivencia sus propias experiencias. Un currículum de vidas se percibe, se habita, se siente. Dado que la experiencia figura al comienzo y al final de la indagación sobre el currículum, las biografías y las identidades forman parte de éste (Rosiek \& Clandinin, 2016).

En el currículum de vidas de la formación docente recuperamos el concepto del conocimiento práctico personal, compuesto de experiencias de vida privadas y profesionales. Cada habitante del aula lo 'porta' activamente, en su carácter moral y emocional. La vivencia de este conocimiento está ligada a narrativas de vidas, nuevamente personales y profesionales (Clandinin, 2019). El 
propio conocimiento deviene narrativo: tiene imágenes, principios prácticos, filosofías personales, metáforas, unidades narrativas, ritmos y ciclos y se define en los siguientes términos:

El conocimiento práctico personal reside en la experiencia pasada del docente, en su mente y cuerpo actuales y en sus acciones y planes futuros. El conocimiento práctico se encuentra en la práctica del docente. Es, para cualquier docente, su manera particular de reconstruir el pasado y las intenciones futuras para enfrentar las exigencias de la situación presente. (Connelly \& Clandinin, 1988, p. 25)

Dicho conocimiento se reencarna y se co-construye una y otra vez en relatos de vida docentes, en contextos definidos por la metáfora del paisaje de conocimiento profesional. Éste comprende las "relaciones entre personas, lugares y cosas" de carácter "moral e intelectual" (Clandinin \& Connelly, 1995, pp. 4-5). De esta manera, es posible transitar e indagar narrativamente el currículum de vidas en la formación inicial del profesorado para recuperar su conocimiento práctico personal acerca de qué significa ser y devenir un buen docente. Dichos s(ab)eres moran en la continuidad de las experiencias vitales de profesores en ciernes y emergen en las narrativas del conocimiento práctico personal. Constituyen asimismo una invitación a comprometerse con lo único de nuestras existencias (Osberg \& Biesta, 2021)

\section{La narrativa como indagación y pedagogía}

La indagación narrativa conceptualiza narrativamente al ser y al devenir de la experiencia, así como a las formas de conocerla (Clandinin, 2016). En tanto metodología y práctica se compromete a vivir, contar, volver a contar y volver a vivir experiencias en la formación del profesorado. Así, como ontología y epistemología, ahonda en el 'conoser' de la experiencia relatada (Clandinin \& Murphy, 2009). La indagación narrativa constituye una pedagogía narrativa porque estudia y recoge relatos de esa experiencia que se crea narrativamente en el currículum. Éste resulta, como hemos manifestado, "un transcurso de vida, ... un viaje que continuamente emerge y cobra forma a lo largo de su recorrido" (Huber et al., 2014). Al contar y volver a contar la experiencia como pedagogía, se piensa con ella y a través de ella: no sólo vivimos experiencias, sino que vivimos relatos de esas experiencias (Contreras Domingo, Quiles-Fernández, \& Paredes Santín, 2019).

Nuestra indagación narrativa reside en el contexto del Profesorado de Inglés de la Universidad Nacional de Mar del Plata, Argentina, en cuyas aulas transitamos los autores como docentes-investigadores. Nuestro grupo de participantes se halla integrado por 24 estudiantes cursantes de una asignatura cuatrimestral de segundo año del área curricular de habilidades lingüísticas del Profesorado de Inglés, quienes escogieron los siguientes seudónimos: Ant, Cas, Clara, Coty, Emma, Fátima, Grian, Heaven, James Nicholas White, Jo, Juana, Lily, Maggie, Marilyn, Married, Polka Dots, Rose, Rusa, Savannah, Sheila Tarnosky de Polasia, Sofía, Tute, Unnamed y Victoria.

Durante las clases de la asignatura, para compartir los relatos de estos futuros docentes en torno a experiencias de su formación, tejimos dialógicamente historias para poder indagar narrativamente en forma oral y escrita sobre: biografías escolares, universitarias y lingüísticas; profesores memorables; desafíos superados; ensayos identitarios; y momentos decisivos. También efectuamos comunicaciones y diálogos variados entre investiga- dores y participantes para profundizar las narrativas estudiantiles y clarificar las opiniones de nuestro grupo. Estos ejercicios en la pedagogía narrativa universitaria nos permitieron brindar atención a las experiencias vividas de este conjunto de docentes en ciernes (Quiles-Fernández \& Martínez, 2019).

Nuestro abordaje de las historias recogidas es completamente narrativo, primero mediante procesos de co-composición y aclaración con cada estudiante de sus relatos sobre los temas planteados. Fue así que urdimos tramas narrativas grupales densas (Caine \& Steeves, 2009), que comportaron un entretejido polifónico de 'hebras' de significados de citas textuales de las 24 voces estudiantiles. Esta forma de aproximarnos a las narrativas compartidas posibilitó la 'cristalización' de experiencias relatadas con luces y sombras, aseveraciones y dudas, cuando armonizamos "simetría y sustancia con una variedad infinita de formas, sustancias, trasmutaciones, multi dimensiones y ángulos de enfoque" (Richardson \& St. Pierre, 2005, p. 963).

Seguidamente, debido a la extensión del trabajo, iniciamos nuestro entramado narrativo coral donde emergen, a partir de las vidas estudiantiles co-relatadas, sus experiencias con docentes memorables a lo largo de todas sus biografías escolares.

\section{Relatos hilvanados de ser y devenir buen docente}

La docencia memorable en muchas asignaturas ha trazado profundas huellas en las vidas del grupo de participantes. ${ }^{1}$ Sheila rememora a su maestra de inglés y español de primer grado: “era sensible y amable... No le preocupaba tanto que habláramos inglés perfectamente, estaba interesada en enseñarnos lecciones morales". Lily y Rusa, a quienes les desagradaba Matemática, señalan a dos profesoras de la materia que ellas comprendieron y disfrutaron sólo con estas docentes. La profesora de Lily era "apasionada" y "hablaba de los números con mucha alegría y un interés... profundo... resulta muy claro ahora que es uno de mis modelos". Rusa indica que la suya:

estaba siempre de buen humor, era apasionada... me transmitió su pasión porque ésa fue la primera y única vez que disfruté de aprender Matemática. Es fácil que nos guste alguien que da una materia que disfrutamos. Sin embargo, como soy muy mala... pienso que esta profesora fue la mejor, al ayudarme a que los números me resultaran interesantes.

El profesor de Química de Sheila "nos enseñó que la única manera de triunfar era pensar y decidir si lo que nos enseñan es correcto o no". James valora de su profesor de Física el haberle "enseñado cómo pensar. Desde ese momento, si alguna vez oíamos algo que no tenía sentido inmediato, seríamos capaces de debatirlo".

Jo distingue a su profesora de Historia y Sociología: “sabía mucho... Además, sabía compartir ese conocimiento. Explicaba cada tema varias veces... usaba... diferentes estrategias para explicar. También era exigente". Sofía recuerda a su profesor de Historia que enseñaba Sociología: “dejó una marca en mí... admiro plenamente su fortaleza, su compromiso y su pasión por su trabajo". Ant prosigue con su docente de Historia: "gracias a esa profesora, me empezó a gustar la Historia y empecé a entenderla. Comprendí que no había una manera definitiva de enseñar... [pero] a veces no se dan cuenta de todas las herramientas que tienen a mano".

Siguen una profesora de Historia y una de Literatura de Fátima. Las dos se caracterizaban por su buen humor, sus recursos

Los relatos de los estudiantes se transcriben en español rioplatense, su lengua materna. 
y el aliento a la participación: "tuvieron un gran impacto en mi vida. Las recuerdo con cariño y me gustaría agradecerles por su trabajo duro, su dedicación y el amor que pusieron en sus clases". Unnamed incluye a su profesor de Literatura: "un profesional destacado en su área... Su estilo era bastante teatral, porque parecía representar una obra corta cada vez que explicaba algo". Married aludió a su memorable varias veces: "soy... raro porque este profesor que a mí me encantó en la secundaria... Éramos los reclutas... también lo tuve como el ejemplo de mi viejo, siempre una persona muy disciplinar". Rose habla de la arquitecta y profesora de Estética que les enseñaba teoría relacionada con la vida real, incitándolos a dibujar y mostrando sus producciones y fotos.

Fuera de la escuela, Grian escoge a su mentor de arte: "ha habido un gran debate sobre lo que hace 'grande' a cada docente. Dicen el conocimiento, dicen la pasión, pero yo creo que es la humildad". De la misma manera, explica que desea:

tratar a mis estudiantes... con humildad y respeto. Sé que la presunción no me lleva a ningún lado y que si quiero crear un lazo significativo con mis alumnos debo hacerles saber que son valiosos. El solo hecho de ser profesora no me hace ser mejor y voy a aprender de mis alumnos tanto como ellos aprenden de mí.

Haven elige a su maestro de canto: "es capaz de sacar a relucir lo mejor de cualquiera y es la prueba viviente de que los sueños sí se hacen realidad si una trabaja suficientemente duro... Por él sigo en el Profesorado". Emma refiere a su instructor de Taekwondo: "un estilo de vida... Las cosas que él te va diciendo las podés aplicar en... el trabajo, en la vida, en la Facultad... Me hace ver bien las prioridades otra vez".

En las narrativas sobre memorables de la asignatura inglés en la escuela, Savannah cuenta que tuvo a su profesora durante ocho años: "la enseñanza es amor y pasión y mi profesora de Inglés Mariela llena esos dos requisitos". Piensa que el conjunto de docentes escolares "son extremadamente importantes porque sirven como ejemplos de lo que queremos y no queremos ser en el futuro". Cas evoca a su profesora de noveno y tutora: "no sólo me ayudó a ser más fluida, sino que también contribuyó a mi autoconfianza para expresarme en inglés... es parcialmente la razón por la cual llegué a pensar en llegar a ser una profesora”. Maggie estuvo con su profesora cuatro años en la escuela: "sus clases eran interesantes y las aproveché mucho. Lo más importante, me dio consejos desinteresados... No sólo deben dar grandes clases sino también apoyar a sus estudiantes". Una profesora del instituto de Coty "rompía el hielo en la clase con su personalidad cálida", ayudándola porque "era chica y tímida".

Entre grandes docentes del Profesorado de Inglés, Clara y Tute eligen a una profesora dispuesta, guía atenta, esforzada, respetuosa, apasionada, favorecedora del aprendizaje; clara, flexible y tolerante. Esta profesora del área curricular de habilidades lingüísticas, "ha tenido un impacto tremendo en mis métodos de enseñanza y mis creencias". Asimismo, se trata de una "docente sobresaliente" a causa de su "personalidad y actitud frente a sus estudiantes" (Tute). Sus clases "me ayudaron no sólo a mejorar mis habilidades escritas y orales sino también a darme cuenta de la clase de docente que querría ser en el futuro. Realmente admiro su paciencia y compromiso con sus estudiantes" (Clara). Marilyn escoge a una docente del área cultural, pues ambas "hacemos gestos cuando hablamos, ambas decimos lo que pensamos directamente y somos espontáneas... somos exigentes; esperamos mucho del resto y en especial de nosotras mismas. Me hace acordar a mi abuela".
Muchas vivencias poderosas de aprendizaje acontecieron fuera del aula. Tres 'docentes' notables enseñan el currículum de la vida. La madre de Polka es "una persona que no tiene el título pero que me ha enseñado algunas de las cosas más importantes en la vida". Para Victoria, "mi familia ha sido un factor crucial en mis experiencias de aprendizaje... crearon esos momentos de aprendizaje con amor y paciencia". Juana mencionó a una humilde escuela de frontera donde acudió de adolescente: "vivir en Corrientes definitivamente me enseñó mucho. Conocí gente increíble cuyas realidades eran muy diferentes de las mías... Estoy muy agradecida por haber vivido esa experiencia y espero que sus enseñanzas me acompañen el resto de mi vida".

Luego de compartir estos relatos en clase, dialogamos más extensamente acerca de las características de la buena docencia en inglés. Resulta muy importante la práctica moral: "yo recuerdo más eso, la parte humana, la parte moral, que el conocimiento en sí" (Rusa). Para Savannah, un "buen docente se define por lo moral... cómo esta persona es como persona". Sheila repite el enseñar a ser "buena persona" y a pensar críticamente. También lo hace Maggie:

Creo que sin dudas un buen docente de inglés, como cualquier otro profesional, nunca debe dejar de estudiar, aprender y mejorar. Si bien todos los demás factores que hacen a un buen docente son de suma importancia, en mi opinión, se encuentran en segundo lugar. Por ejemplo, características morales, de la personalidad.

La pasión por la enseñanza transmitida a los estudiantes se distribuye en una variedad de expresiones sobre la buena docencia en la clase de inglés: "pasión por la carrera" (Marilyn) y "pasión por su vocación, por la docencia" (James). Sofía opina con vehemencia que:

un buen docente... debe mostrar, principalmente, pasión [que] se transmitirá en sus estrategias didácticas, porque tratará de hacer que los chicos comprendan de cualquier forma, y en su personalidad, porque estará feliz y dispuesto a la hora de enseñar... Puede ser que un docente sea una excelente persona, agradable y súper carismática, pero si... no le gusta su labor, entonces toda su práctica se verá teñida de mala gana y frustración.

El costado afectivo de los vínculos con la clase también resuena con fuerza para esta disciplina. Según Coty, sus buenos y buenas docentes "pueden establecer una buena relación con sus estudiantes". Polka señala que su "buen docente... llega a sus estudiantes... muestra interés en que... comprendan y se interesen por lo que está enseñando". Fátima coincide: "que haga su trabajo con amor... y no como alguien 'superior"'.

Otras voces señalan al conocimiento pedagógico para enseñar el idioma y sus contenidos. Según Jo, "puede saber mucho, pero si no sabe cómo transmitirlo... no va a significar nada". Para Rose, "debe tener las herramientas didácticas para enseñar. Saber contenido no es sinónimo de docencia". Además, "en la Facultad saben mucho, mucho... Hay quienes no saben cómo manejar su conocimiento. Entonces saben que saben... Hay docentes que saben mucho pero no muy pedagógicamente" (Grian). De acuerdo con Juana:

he tenido demasiados profesores... que son excelentes, que yo sé que son brillantes, pero... tienen un montón de títulos y no me transmiten nada. No entiendo lo que hablan, no se 
preocupan por el curso... Hay que hacer un énfasis, particularmente en la [Universidad] Nacional, en desarrollar todo lo que es la habilidad del docente. Eso es lo que vamos a ser.

De la misma forma, quizá para ejemplificar lo que debería ser la buena enseñanza en inglés, dos participantes optan por aludir a docentes de otras materias en la escuela: "sabían muchísimo pero no sabían transmitirlo. El que daba muy buenas clases era el de Literatura que nombré" (Unnamed). Igualmente:

la de Matemática que hizo que me gustara la matemática, yo no entendía nada y... le encontró la vuelta para que a sus estudiantes les gustara. Después también la de Historia... haciendo que se compenetraran en lo que estaban haciendo. (Ant)

Otros relatos aluden al conocimiento disciplinar requerido. Para aludir a la buena docencia en el área de inglés, Haven realiza una analogía con su maestro de canto:

Mi profe se formó rigurosamente, él canta... profesionalmente desde que tiene quince, pero él fue al conservatorio, después se cambió a [un instituto]. Muchísimos años estudiando y estudiando y vos lo ves y él siempre se está perfeccionando, se fue a Buenos Aires y estuvo tomando cursos con gente que ha venido de Estados Unidos. Él sabe las cosas, no te dice algo por decírtelo, él te lo va a decir porque sabe que te va a servir. Incluso me ha dado un módulo de teoría para leer, qué sé yo y hace poco dio un seminario para todos nosotros para que veamos las cuerdas vocales, nos dio ejercicios. Me dijo esto lo aprendí acá y lo trajo tal persona. [En la enseñanza del inglés en las escuelas] noto que es muy repetitivo.

A la par, Emma ofrece una similitud con su instructor de Taekwondo:

no he tenido una figura tan fuerte de inglés. Fue mucho más fuerte la de mi maestro. Lo veo, lo voy a visitar y todo... Un montón de cualidades... El Taekwondo es un estilo de vida ¿no? Entonces todas las cosas que él te va diciendo las podés aplicar en tantos campos, digamos. La puedo aplicar en el trabajo, en la vida, en la facultad, en... no sé. Yo voy y hablo con él y él es como que me ordena de vuelta y me hace ver bien las prioridades otra vez. Porque a veces yo llego muy pasada de rosca. Muy acelerada y me pone mal y la facultad. Entonces él me dice: 'bueno, primero, la facultad, recíbase...' Y todo así como que me ordena de vuelta. Me hace volver.

En otro orden, para Victoria, “cuando era más joven, era para mí más importante la personalidad y los aspectos didácticos del docente [de inglés]. Quizás hoy en día me impacta más el conocimiento disciplinar y la pasión que transmite". Algunas narrativas tratan de hallar un equilibrio entre lo moral, lo disciplinar y lo didáctico: un buen docente "presenta una combinación de buenas características que te dan admiración y querer seguir sus pasos" (Clara). Según Tute, es una combinación de lo moral y lo disciplinar que "no sólo me genera admiración sino curiosidad porque "le puedo preguntar". Es un "equilibrio": tener "conocimientos de los temas..., interés personal por enseñar y aprender constantemente, ... paciencia, ponerse en el lugar del resto..., seguir investigando" (Cas). A Lily le resulta difícil explicar qué hace a la buena docencia en inglés. Según Married, las cualidades ideales varían según los contextos: en el colegio, memorables eran gamba, copados. En la Universidad... van muy bien en la parte disciplinar como en el trato con sus estudiantes, tienen mucha experiencia para compartir y te pueden ayudar a formar como humano. Tal vez el modelo en los institutos de inglés... es algo desestructurado, que sabe mucho del idioma, que ha viajado.

En un cierre provisorio de significados, hallamos trazas de conocimiento pedagógico-disciplinar para enseñar utilizando diversos materiales y medios. También notamos estrategias de enseñanza y de razonamiento pedagógico para transformar contenidos y materiales en potentes intervenciones didácticas. Finalmente, se destacan los actos morales, así como la experticia universitaria.

\section{Reflexiones emergentes de las hebras narrativas en torno a los buenos docentes}

Nuestra indagación se interroga sobre las formas en que se entraman experiencias vividas en, y con, la buena docencia mediante relatos compartidos en la formación inicial de nuestros participantes-estudiantes del profesorado de inglés. Al respecto, los hilos narrativos que tejimos sugieren fuertemente que el currículum de la formación docente no refiere sólo al documento formal denominado Plan de Estudios del Profesorado de Inglés, sino que incorpora múltiples docentes como co-protagonistas y co-guionistas con quienes el grupo de participantes representan y co-componen sus experiencias de aprender a enseñar. Estos relatos sobre buena enseñanza integran un currículum para s(ab)er y 'haser-se-r' docente que "está poblado, superpoblado, con las intenciones de" personajes y coautorías (Bakhtin, 1994, pp. 293294) con quienes se han co-forjado narrativas sobre quiénes (no) se desea ser como docentes.

Estas experiencias vividas sobre la buena enseñanza y los buenos docentes a lo largo de la vida escolar que recogimos se ubican entre las relaciones sociales y sus interpretaciones, entre memorias y sucesivas reencarnaciones. Las narrativas recuperan no sólo lo que sucedió en la buena docencia, lo que los docentes memorables hicieron, sino también lo que no ocurrió, no debiera haber ocurrido o lo que algunos docentes no realizaron o no debieron haber realizado. Con su contigencia y su puesta en acto dialógica, estas narrativas llegan al corazón de la docencia, que no es individual sino relacional y capaz de resignificarse.

Nuestros relatos sobre las vivencias en torno a la buena docencia y los buenos profesores no son algo recibido que simplemente escuchamos. Por el contrario, son algo que nos crea, que hace a quiénes devenimos. A la sazón, aprender a enseñar es narrar relatos de lo que esa experiencia nos hace. Se trata de "una búsqueda de significado y una esperanza de que la experiencia docente transforme al significado en discernimiento". De esta manera, "aprender a enseñar es un problema de significación y una actividad interpretativa" (Britzman, 2003, p. 19). Implica aprender a devenir porque nadie nos brinda la experiencia o el significado. En esta búsqueda, creamos relatos propios y ajenos: surgen infinitos relatos de aprender a enseñar.

Nuestra indagación narrativa y su pedagogía narrativa revelan currícula propios que el grupo de estudiantes construyó para su formación y que comprende variados dispositivos: "aquellos espacios, mecanismos, engranajes o procesos que facilitan, favorecen o pueden ser utilizados para la concreción de un proyecto o la resolución de problemáticas" (Sanjurjo, 2009, p. 32). Las narrativas sobre la buena enseñanza y los buenos docentes: 
se constituyen en memoria como saber de la experiencia desde la búsqueda de trazos que incluyen el recuerdo de lo imprevisible, la perplejidad, los límites; de un saber en perspectiva que se asienta sobre el relato que enfatiza la presencia insoslayable de la subjetividad, de la contingencia de cada trayecto; de un movimiento en el que el sujeto reencuentra los sentidos que inciden en su presente. (Davini, 2002, p. 73)

Así, cada docente en formación deviene un relato vivo en sus "paisajes relatados", donde su existencia se entrelaza con los contextos donde compone su s(ab)er y su devenir. Éstos constituyen "la encarnación única de sus relatos para vivir, relatos formados por los paisajes presentes y futuros donde habita y trabaja" (Clandinin \& Huber, 2005, p. 44). Nuestros estudiantes detentan grandes caudales experienciales aprendidos en el transcurso de sus currícula de vidas, transitados en paralelo a los currícula escolares y universitarios formales. En esta dirección, los significados que emergen de nuestras hebras textuales se vinculan con el espíritu de la indagación y la pedagogía narrativas. Todas las personas son múltiples relatos: el currículum para la formación docente debe incluir el sinnúmero de nuestras historias individuales y grupales (Caine, Estefan \& Clandinin, 2013).

Las hebras tejidas de estas voces de docentes en ciernes no simplemente visibilizan su conocimiento práctico personal (Clandinin, 2019; Connelly \& Clandinin, 1988). El entramado de relatos en el gran tapiz narrativo que co-urdimos nos permite co-autorías y co-interpretaciones de las experiencias, las teorías y las prácticas que vamos co-forjando, en lugar de meras repeticiones de teorías o habilidades recibidas. En esta reconfiguración de lo privado en la esfera pública del aula, abandonamos la matriz reproductora de la formación del profesorado, dilatadamente criticada pero aún vigente. (Liston \& Zeichner, 1997). Nuestro grupo de estudiantes-futuros docentes co-produjo y co-representó conocimientos a partir de sus vidas, desplazándose allende el currículum como instrumento externo y normado socio políticamente en su teleología y arribando a un currículum con conocimientos más (auto)emergentes, abiertos y continuos (Osberg \& Biesta, 2021).

\section{Conclusiones}

De nuestros entramados narrativos surgen experiencias vividas sobre el s(ab)er de la buena docencia que inscriben nuestras posibles contribuciones dentro de la indagación y la pedagogía narrativas como metodologías íntimas de exploración para formación docente (Pinnegar \& Hamilton, 2017). Se trata de prácticas privadas que recuperan, fundamentalmente, conocimientos prácticos personales. La intimidad encierra también el compromiso de quienes indagamos y participamos activa, comprometida e 'interesadamente' de nuestros propios contextos, porque la enseñanza y el aprendizaje son actos privados de carácter público.

En cierto modo, efectuamos una "ruptura con la agenda convencional y canónica de la formación de profesores y desafían el estatus de legitimidad conferido a algunos tipos de saberes y sus pretensiones de verdad científica". Podemos decir que hemos "dejado de manifiesto una suerte de currículum paralelo, fuertemente emocional y distintivamente personal y humano, que atraviesa la construcción de las biografías escolares y es altamente pregnante en la configuración de las identidades". Nuestra indagación narrativa constituyó un "modo privilegiado de acceso a una sabiduría ubicua e irrefutable que se resiste a alojarse en los textos y las prácticas académicas convencionales" (Suárez \& Dávila, 2018, p. 358). Nuestro trabajo acuerda, entonces, con las propuestas de abandonar al currículum como instrumento externo o normado socio políticamente en su teleología (Osberg \& Biesta, 2021). Ontológicamente, la educación es un fenómeno que nace, que es (auto)emergente, abierto y continuo. Más allá de servir su propósito, crea ese fin único al cual sirve, pues la estructura y el propósito son inseparables de aquello que surge. En este sentido, un auténtico currículum de vidas brotó de nuestra indagación y de nuestra pedagogía narrativa.

A la sazón, experimentar el inmenso privilegio de cultivar y velar por estos 'pequeños grandes' relatos que nos fueron confiados significó asomarse a la excepcional riqueza de las experiencias que se iban develando con el correr de la indagación. Como profesores-investigadores y formadores de docentes, sentimos que las narrativas de estos seres, que devienen tanto en su mismidad como en su otredad, revelan los entrecruzamientos del curriculum de vidas como recorrido existencial y el currículum académico-formal en los cuales dichas existencias toman paralelamente sus caminos educativos.

\section{Referencias}

Apple, M. (1986). Ideología y currículum. Madrid: Akal.

Bourdieu, P. (2003). Capital cultural, escuela y espacio social. Buenos Aires: Siglo XXI.

Britzman, D. P. (2003). Practice makes practice. A critical study of learning to teach. Revised edition. New York: State University of New York Press.

Caine, V., Estefan, A., \& Clandinin, D. J. (2013). A return to methodological commitment: Reflections on narrative inquiry. Scandinavian Journal of Educational Research, 57(6), 574-586.

Caine, V., \& Steeves, P. (2009). Imagining and playfulness in narrative inquiry. International Journal of Education $\mathcal{E}$ the Arts, 10(25), n25.

Connelly, F.M. \& Clandinin, D.J. (1988). Teachers as curriculum planners: Narratives of experience. New York: Teachers College Press.

Contreras Domingo, J., Quiles-Fernández, E., \& Paredes Santín, A. (2019). Una pedagogía narrativa para la formación del profesorado. Márgenes, Revista de Educación de la Universidad de Málaga, 0(0), 58-75.

Clandinin, D. J. (2016). Engaging in narrative inquiry. London-New York: Routledge.

Clandinin, D. J. (2019). Journeys in narrative inquiry: The selected works of D. Jean Clandinin. New York: Routledge.

Clandinin, D. J., \& Connelly, F. M. (1995). Teachers' professional knowledge landscapes. New York: Teachers College Press.

Clandinin, D. J., \& Huber, M. (2005). Shifting stories to live by. In D. Beijaard, P. C. Meijer, G. Morine-Dershimer, \& T. Harm (Eds.), Teacher professional development in changing conditions (pp. 43-59). Dordrecht: Springer.

Clandinin, D. J., \& Murphy, M. S. (2009). Comments on Coulter and Smith: Relational ontological commitments in narrative research. Educational Researcher, 38(8), 598-602.

Davini, M. C. (2002). La iniciación en las prácticas docentes en las escuelas. En M. C. Davini (Coord.), De aprendices a maestros. Enseñar y aprender a enseñar (pp. 13-38). Buenos Aires: Papers Editores.

Da Silva, T. T. (2001). Espacios de identidad: nuevas visiones sobre el currículum. Barcelona: Octaedro.

De Alba, A. (1998). Currículum: Crisis, mito y perspectivas. Buenos Aires: Miño \& Dávila.

Dewey J. (1998). Experience and education. Indianapolis, IN: Kappa Delta Pi.

Eisner, E. W. (1994). Cognición y currículum. Buenos Aires: Amorrortu. 
Freire, P. (1970). Pedagogía del oprimido. México, D.F.: Siglo XXI.

Gimeno Sacristán, J. (1998). El currículum: una reflexión sobre la práctica. Madrid: Morata.

Huber, J., Caine, V., Huber, M., \& Steeves, P. (2014). La indagación narrativa como pedagogía en la educación: el potencial extraordinario de vivir, contar, volver a contar y revivir relatos de experiencias. Revista de Educación, 7(5): 33-74.

Jackson, P. W. (1968). La vida en las aulas. Madrid: Paideia-Morata.

Liston, D. P., \& Zeichner, K. M. (1997). Formación del profesorado y condiciones sociales de la escolarización. Madrid: Morata.

Magrini, J. M. (2017). Dwelling and creative imagination in Gaston Bachelard's phenomenology: Returning to the poetic space of education and learning. Educational Philosophy and Theory, 49(8), 759-775.

Osberg, D., \& Biesta, G. (2021). Beyond curriculum: Groundwork for a non-instrumental theory of education. Educational Philosophy and Theory, 53(1), 57-70.

Pinnegar, S., \& Hamilton, M. L. (2017). The promise of the particular in research with teacher educators. In D. J. Clandinin \& J. Husu (Eds.), The SAGE handbook of research on teacher education (pp. 1065-1080). Thousand Oaks, CA: SAGE.

Popkewitz, T. S. (2010). Alquimia y gobierno, o preguntas acerca de lo que preguntamos. Revista de Educación, 1(1), 15-41.

Quiles-Fernández, E., \& Martínez, S. O. (2019). Atender las tensiones educativas en la formación inicial del profesorado: La indagación narrativa como práctica pedagógica. Revista Interuniversitaria de Formación del Profesorado, 33(3), 105-120.
Richardson, L. \& St. Pierre, E. A. (2005). Writing. A method of inquiry. In N. K. Denzin \& Y. S. Lincoln (Eds.), The SAGE handbook of qualitative research (959-978). Thousand Oaks, CA: SAGE.

Rosiek, J., \& Clandinin, D. J. (2016). Curriculum and teacher development. In D. Wyse, L. Hayward, \& J. Pandya (Eds.), The SAGE handbook of curriculum, pedagogy and assessment, 1 (pp.293-308). Los Angeles-London: SAGE.

Sanjurjo, L. O. (2009). Razones que fundamentan nuestra mirada acerca de la formación en las prácticas. En L. O. Sanjurjo (Coord.), Los dispositivos para la formación en las prácticas profesionales (pp. 15-43). Rosario, Argentina: Homo Sapiens.

Santos Guerra, M. A. (2008). La pedagogía contra Frankenstein. Barcelona: Grao.

Seiki, S., Caine, V., \& Huber, J. (2018). Narrative inquiry as a social justice practice. Multicultural Education, 26(1), 11-16.

Shulman, L. (1986). Those who understand: Knowledge growth in teaching. Educational Researcher, 15(2), 4-14.

Stenberg, K., Karlsson, L., Pitkaniemi, H., \& Maaranen, K. (2014). Beginning student teachers' teacher identities based on their practical theories. European Journal of Teacher Education, 37(2), 204-219.

Suárez, D. H., \& Dávila, P. V. (2018). Documentar la experiencia biográfica y pedagógica. La investigación narrativa y (auto) biográfica en educación en Argentina. Revista Brasileira de Pesquisa (Auto)biográfica, 3(8), 350-373. 
